\title{
THE ROAD TOWARDS MULTI-HYPOTHESIS INTENTION SIMULATION AGENTS ARCHITECTURE - FRACTAL INFORMATION FUSION MODELING
}

\author{
Per M. Gustavsson \\ University of Skövde \\ School of Humanities and Informatics \\ Högskolevägen, Box 408, Skövde, 54128 Sweden
}

\author{
Tomas Planstedt \\ Ericsson \\ Security Solutions \\ Storgatan 20, Skövde, 541 30, Sweden
}

\begin{abstract}
This paper presents the road towards Multi-Hypothesis Intention Simulation Agents Architecture and is focused on the Fractal Information Fusion model (FIF) that are formed to support a systems-thinking in an agent architecture that aligns with the Global information Grid, NATO Net Enabled Capabilities and Swedish Armed Force Enterprise Architecture initiatives. The Joint Directors of Laboratories information fusion model and the Observe, Orient, Decide, Act loop by John Boyd is combined and used as the foundation together with the Knowledge Model, Level of Conceptual Interoperability shaping the FIF-model. The FIFmodel's effect in shaping of the Multi-Hypothesis Intention Simulation Agents Architecture is presented.
\end{abstract}

\section{INTRODUCTION}

The Global Information Grid (GIG), the NATO Net Enabled Capabilities (NNEC) and the Swedish Armed Force Enterprise Architecture (FMA) challenges the Command \& Control, Communications, Computers and Intelligence (C4I) systems of today. The GIG, NNEC and FMA are all built on system views containing the perspectives, Enterprise Services (capabilities), Enterprise Systems, Enterprise Functions, and Enterprise ability organized as a Capability Package (CP) with Technical-, Information- and Personnel components (United States. Joint Chiefs of Staff. 2000; Försvarsmakten Högkvarteret. 2003; Öhlund et al. 2003; Buckman 2004). In order to achieve interoperability in reality as well as in the simulation domain the trend is that implementations based on Service Oriented Architecture (SOA) will enable the interoperability needed (Tolk et al. 2003a; Tolk et al. 2003c; Gustavsson et al. 2004).

The net-centric SOA interoperability paradigm will lead to the ability to connect systems, technical and/or human, together in new larger constellations than ever before. This will probably also signify that providers and consumers will use the capability of connecting systems together enabling the future Consultation, Command \& Control
(C3I) systems. There is no doubt that more systems will generate more data flowing in the technical-net. In order to get information and knowledge out of all this data efficient methods of aggregating, fusion and interpreter the data into information are in deed needed.

The one with the most efficient Observe, Orient, Decide, Act (OODA) loop will gain and preserve Information Superiority (IS) and Full Spectrum Dominance (FSD), presented in (United States. Joint Chiefs of Staff. 2000). The battle in it self does not necessary be in the military domain, the battle or struggle could be in the large domain of Operation Others Than War (OOTW).

To truly achieve the IS and FSD the opponents intention need to be assumed in some way by the user of a C3I system. The opponent do have a plan, the complexity is that the old fashioned cold-war scenario does not apply anymore. There will not be static doctrines that a decision-maker can map situations against. The need for a more dynamically approach is necessary in manage doctrines. In "Joint Vision 2020" and "Målbild för försvarsmaktens ledningssystem" (United States. Joint Chiefs of Staff.; United States. Joint Chiefs of Staff. 2000; Försvarsmakten Högkvarteret. 2003) the picture is given that the technology change will impact on the current doctrines and according to the (Defense Material Administration 2003) constantly change is the only constant state, meaning that the only thing a decision maker can be sure off is that the next time doing the same act the opponent has changed tactics.

The DSS is often built upon knowledge from known doctrines and how they are used. The observed and assumed doctrines of our opponent are fairly ruff these days. Back in the cold war era intelligence had a much easier task in gathering information. Some monitoring from battle training maneuvers resulted in updates in the doctrines. The forces looked the same the ideological doctrines well known. Today a decision maker is facing a new situation where the mind of the opponent is often unknown, and when decision maker learns the behavior the opponent adopt and uses another doctrine. In this world there is not one doctrine, one overall hypothesis, there are several. To get hold of the op- 
ponents intention approaches using Multi-Hypothesis resolving is emerging. Examples are the "Multi-Hypothesis Database for Large-Scale Data Fusion" (McDaniel 2002), the Combat ID approach in "Multi-Hypothesis Structures, Taxonomies and Recognition of Tactical Elements for Combat Identification Fusion" (Schuck et al. 2004) and "MultiHypothesis Abductive Reasoning" in (Pioch et al.) Just to mention a few discussing multi-hypothesis generating and resolving.

The paper starts with a foundation part giving an overview of some topics relevant for the paper: OODA-loop, decision-making, information fusion. Continues with motivation part describing the environment for the decision maker. The FIF-model is introduced and then the MultiHypothesis Intention Simulation Agents novel architecture is presented before the summarization.

\section{FOUNDATION}

The ability to interact with the environment and those within it in an appropriate fashion is essential. But without knowledge, the outermost necessity when making decisions, decision-making is blindfolded. The ability to observe and orient and where possible shaping the environment or else adapting to it makes the decision maker prosper. In this section the authors give the foundation for the work with the FIF-model.

\subsection{OODA-loop}

The traditional way of looking at a decision cycle is to use the loop defined in the presentation of "Patterns of Conflict" (Boyd 1986), Observe, Orient, Decide, Act (OODA) viewed in (Figure 1), here somewhat relaxed. Regardless if the character is friendly, enemy, noncombatants they all share that every day in every moment the mind deals with tasks of commonplace to the outermost complicated. In the process observing the surrounding environment, building hypothesis, weight plans towards each other, and then hopefully be proactive not reactive, thereby improving the chances of kept or entailed prosperity. An OODA-loop. plored.

The OODA-loop definition used by the authors ex-

Observe The starting blocks, obtain sensor ${ }^{1}$ information i.e., find the threat before he or she finds you.

Orient Establishing reality, put things in proper perspective based on real time input, previous intelligence, and generated assumptions.

Decide The pipeline, reasoning of alternatives creating a matrix of actions

\footnotetext{
${ }^{1}$ Sensor in this context implies both human and nonhuman
}

Act Performing move; carry out actions from the matrix.

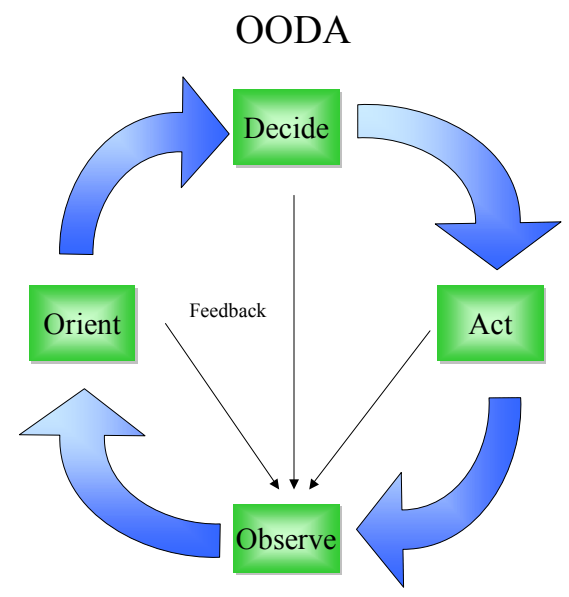

Figure 1: OODA Loop by John Boyd

Worth noticing is that from each step feedback is sent to the process of gathering more/better/refined observations refining the other steps further entailing and maintaining freedom of action.

To really take full advantage of the OODA-loop, the humans, using systems, require training. The training needs to cover the whole OODA-loop enabling for the mind to recognize previous situations. Then the reacting will be done in harmony with comfort and ease showing proactive and award full spectrum dominance.

\subsection{Decision Making}

Making decisions is a non-trivial task to accomplish. When taking a glance at decision making in practice. As a human we can, from our self, divide the decision process into two paths: The conscious mind which works in serial or sequentially, handling seven plus minus two variables before taking no notice of or misinterpreting incoming data; On the other hand the subconscious mind which can process hundreds of variables simultaneously, in parallel. So how to achieve a good support in decision-making? One way is through training, i.e. simulating the tasks that are arising with alternatives that can arise doing it. This is not new, all training maneuvers, the whole I/ITSEC exhibition and the most obvious of them all, School, to just mention the observable fact of training.

Just go to your self when driving a car and suddenly a ball appears in the windscreen view of yours. Do you really process all your alternatives searching for the best decision to act upon; can you really honestly say that you thought? Actually you react, and if you avoided the child tumbling out after the ball you will probably say that you had good luck. Stated in the beginning there are two process lines in the mind. In the above situation the subconscious mind where acting, etching small data tokens in 


\section{Gustavsson and Planstedt}

your memory of the incident: the ball is bumping out; you looking at the side for the additional kid; Seeing the kid; During the whole phase constantly breaking and steering away from the direction of the ball. Hey, you just walked through the OODA-loop as well as a multi-sensor information fusion process relying on your inbuilt active controlling decision support system (DSS).

That's impressing, but the next time you encounter a similar situation you will be less stressed acting more precise with a gainful outcome. You have learned the data fragments are identified, sorted, stored and are ready for retrieval and use. The mind discovers new courses of action perform experimentations ending up with Knowledge.

\subsection{Decision Support Systems}

Decision support is a complex task and the authors believe that there will never ever be a system that can replace the mind of humans. However the authors are truly convinced that computer based support systems can challenge the human mind and even some times do the decisions for the human. As an example the Deep Blue (IBM 1997) showed that it at least is possible to create decision systems that can play chess better than the best human. In environments that are complex and full of dynamic behavior, some kind of support to ease the human decision burden is certainly appropriate. Still one must not forget that the human is consulting, commanding and controlling the system ${ }^{2}$ and takes the decision and makes the action.

Decision-making could be divided in to three major activities: planning, directing, and monitoring. Adopting this three phases to the OODA-loop is quite easily done. Planning maps to Decide, Directing to Act and Monitoring to Observe and Orient phase. The main difference is when you enter the loop, planning or observing. Therefore the authors use the OODA-loop since it entails the plan, direct and monitor structure Many DSS are built upon the OODA-Loop and the plan, direct, monitor models. In general terms these systems provide the decision maker with the ability to see more information (Observe and Orient) and tools for Command and control (Act). Automatic decision support is seldom present and when at hand the support is simple, plain, undeveloped yet promising but still so fragile. Just pushing more information at a decision-maker does not lead to making better decisions. And what is information?

\subsubsection{Knowledge, Information and Data}

The author's definitions of data, information and knowledge is as follows:

\footnotetext{
${ }^{2}$ Here the system can contain Personals, Organizations, and Technical-systems together or alone in any configuration.
}

Data is the method of transport and storage expressed in symbols such as numbers, characters, words, sentences, and objects, pictures that are not put into a context.

Information is data that are put into a how, when, what and why context for usage of a specific target (human, non-human). Ex. symbols arranged in a certain style applied on a paper with additional data about coordinates etc. Holds an information piece called a map.

Knowledge is a reproduce of true reality. The information peaces known are combined and evaluated forming knowledge pieces that if they are relevant and reusable creates the ability to knowledge usable in new situations.(Kock 2005)

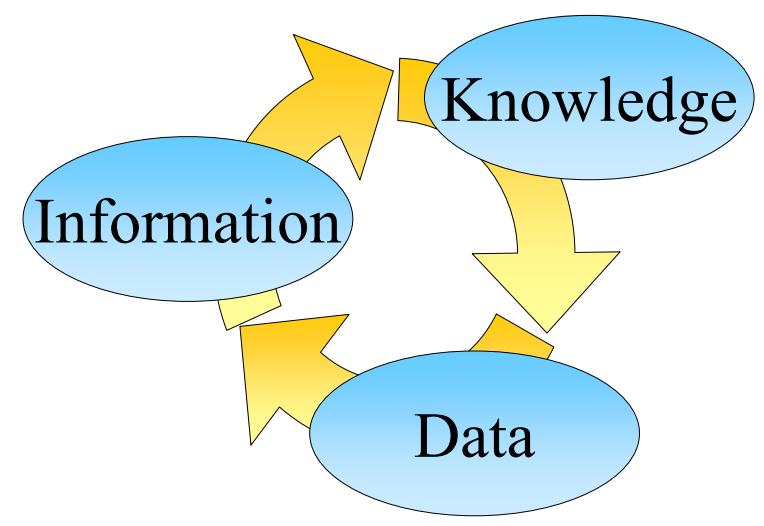

Figure 2: Knowledge Information and Data Model

Data put into a context is then information but if this information occur in another context as well, the information is just data within this new context. Hence, information put out of its context is merely data in that new context but still it is information in the first context. This gives us that a set of data can be both data and information simultaneously; it is up to the system view of the data/information. The same goes for knowledge; knowledge for one system is data/information for another forming a recursive Data/Information/Knowledge model (Figure 2). Often the effort in presenting models are assuming that there is only one instantiation one scope of the model, for example JDL

\subsection{Information Fusion}

The research area of information fusion, which is normally presumed to include the narrower terms data fusion, sensor fusion, image fusion, decision fusion and knowledge fusion, has only emerged relatively recently. As a consequence, the field and much of its terminology are still developing.

There are two main reasons and developments for the steady increase in interest and research activity in information fusion the last decades. First, there is an increasing need for information fusion, filtering or extraction in times 


\section{Gustavsson and Planstedt}

of what many perceive to be a constantly growing information overflow. Second, there is an increase in availability of information owing to, not only technological developments, but to societal developments as well.

A commonly cited statement of the fusion problem is the following quote from Naisbitt (1982) that still characterizes the problem:

We are drowning in information but starved for knowledge. This level of information is clearly impossible to be handled by present means. Uncontrolled and unorganized information is no longer a resource in an information society, instead it becomes the enemy.

The field of information fusion is commonly characterized as a multidisciplinary research area and overlaps with a number of other areas that have their own, partly overlapping, research communities, conferences and journals. It is therefore not surprising that notions and definitions of information fusion range from very broad ones to narrower, application-specific ones, especially in military applications.(Andler et al. 2004)

\subsubsection{JDL}

One way of describing Information fusion is the JDL model which is commonly used within the Defence community. The model has its origin in the works performed by the Department of Defense of the United States of America, and especially by the Data Fusion Sub panel of the Technology Panel for C3 (command, control, and communications) of the Joint Directors of Laboratories (JDL).

The JDL developed a functional model that illustrates the primary functions, relevant information and databases, and interconnectivity to perform data fusion.

A more concise definition was later proposed by (Steinberg et al. 1999) as: data fusion is the process of combining data to refine state estimates and predictions. The composition of each of the levels as follows:

Level 0 - Sub-Object Data Assessment estimation and prediction of signal/object observable states on the basis of pixel/signal level data association and characterization

Level 1 - Object Assessment estimation and prediction of entity states on the basis of observation-to-track association, continuous state estimation (e.g. kinematics) and discrete state estimation (e.g. target type and ID)

Level 2 - Situation Assessment estimation and prediction of relations among entities, to include force structure and cross force relations, communications and perceptual influences, physical context, etc.

Level 3 - Impact Assessment estimation and prediction of effects on situations of planned or estimated/predicted actions by the participants; to include interactions between action plans of multiple players (e.g. assessing susceptibilities and vulnerabilities to esti- mated/predicted threat actions given one's own planned actions)

Level 4 - Process Refinement (an element of Resource Management) adaptive data acquisition and processing to support mission objectives

Since then another extension to the revised JDL model has been suggested this suggestion includes a fifth level.

Level 5 - Human (or User) Refinement which addressed the issues associated with the human interface to and control of the DF process.

The model at this point would thus have 4 core fusion Levels (L0-L3) and 2 extension Levels (L4-L5) (Llinas et. al. 2004)

\subsubsection{Information Fusion Process}

According to (Andler et al. 2004) information fusion is the synergistic integration of information from different sources about the behavior of a particular system (Figure 3 ), to support decisions and actions relating to the system.

Information fusion includes theory, techniques and tools for exploiting the synergy in the information acquired from multiple sources, for example sensors observing system behavior, databases storing knowledge about previous behavior, simulations predicting future behavior and information gathered by humans.

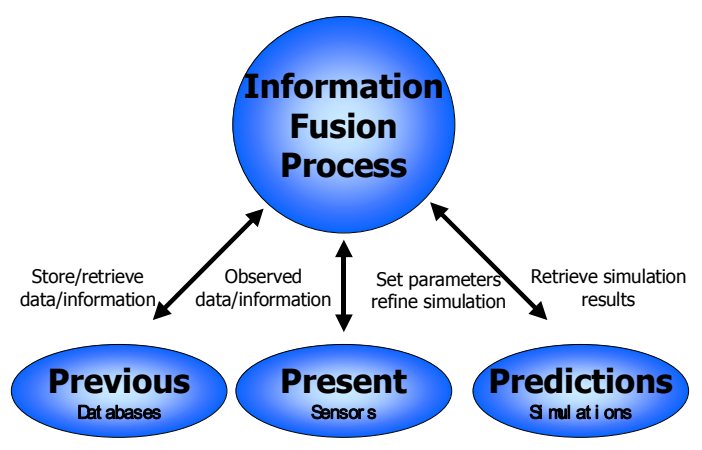

Figure 3: The P3IF process

The resulting decision or action is in some sense better, qualitatively or quantitatively, in terms of accuracy, robustness and comprehensibility, than would be possible if any of these sources were used individually without synergy exploitation. Note that information fusion not only deals with actual fusion processes, but also with how the results of these processes can be used to improve decisionmaking. In other words, the generic context of information fusion is the process of gathering relevant information, fusing different information sources and interpreting the result, making decisions based on the interpretation and acting according to those decisions (Figure 4). 


\section{Gustavsson and Planstedt}

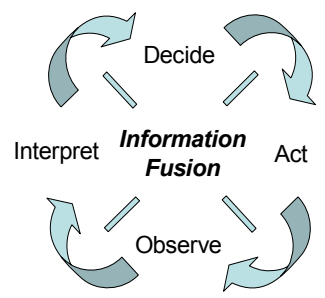

Figure 4: Information fusion in a generic context

\section{MOTIVATION}

So what motivates yet another approach to the ultimate decision support system. The authors argue that there does not exist a computer-based ${ }^{3}$ decision support system that can aid in all kinds of situation that the system might step into. Therefore the work in making such a support system is challenging and with today's enormous capacity of data retrieval, sensors, processors, memory a further step can be made.

\subsection{The GIG, NNEC, FMA}

Within the (United States. Joint Chiefs of Staff.; United States. Joint Chiefs of Staff. 2000; Försvarsmakten Högkvarteret. 2003; Öhlund et al. 2003; Buckman 2004) that describe ideas for the future - or rather preparing for the unknown future. One of the changes for the future is that the forces, systems shall be able of participate in joint constellations. Joint here means joint between services branches, joint across nations and joint between agencies, not necessary military focused. Further Full Spectrum Dominance implies that forces are able to conduct prompt, sustained, and synchronized operations with combinations of forces tailored to specific situations and with access to and freedom to operate in all domains - space, sea, land, air and information.(United States. Joint Chiefs of Staff. 2000). The technology is developing so fast that there is a risk that the ability to capture ideas and formulate operational concepts will be exceeded. The vast amount of information that will pour upon us will also have better quality than today. The capability to collect, process information will be essential. The system of the future needs to be interoperable with each other. Not only at technical level but also at Syntactic, Semantic, Dynamic, Pragmatic and conceptual level (Tolk et al. 2003b; Tolk 2004). The future will be more complex, more dynamic, more noisy and the future systems need to handle this.

\footnotetext{
${ }^{3}$ Computer-based is essential to say since the authors mother and fathers are the best decision support system ever made for them. And hopefully the Childs of the authors will say the same when they mature.
}

\subsection{Change of the Opponents Behavior}

As stated before the opponent looks asymmetric since the ability to find his tactics, doctrine is a hard task to perform. But lets step into the mind of our opponent for a second, $\mathrm{He}$ or she wants to cause the greatest damage to us anywhere any time anyhow. But still, regardless at which side we find our self's to be, bad or good, we regularly do not want to give up our lives, families and why of living. Just that simple assumption decreases the space where they and we can act. Further we all will strike where the opponent are weakest. So why not give our opponent a weak spot. Effect Based Operations (EBO) an old knowledge coming into new discuss how to maneuver your opponent into a state where you have Full Spectrum Dominance.

Underestimating your opponent is the first step to failure, the opponent are at least as good as us in adapting to new situations. The opponent is living in the shared environment with the same education, using the same tools right beside us waiting for the opportunity to strike.

The need for systems that reduces and limits the risk of loss of lives at both sides is essential.

\subsection{Simulation the Key Enabler}

Simulation is the key enabler both in developing systems of the future, finding out which systems ideas that might work in which situations without causing casualties of humans, wasting resources and money. Also simulation is used in training decisions/makers at all levels. Within generating and evaluating intentions, simulation is essential since there will not be time, (processing capability), to go thru all possible cases.

\section{THE FIF MODEL}

Why a new model, is there not enough of models? Yes but the existing models do not encapsulate the whole picture of decision-making and information fusion. The authors believe that the FIF-model approach gives another view that is helpful in the process of developing decision support systems.

\subsection{OODA and JDL}

In the paper SDAFLF by Mark D Bedworth an analogy is made between OODA and JDL where it stated that level-0 (Signal) is the observe part, level 1-2 (Object, Situation) is orient, level-3 (Intention) is decide and level-4 (refinement) is act (Figure 5). But if the decision phase includes finding action matrixes then the step prior must be of intention discovery (threat is one). This approach is adopted in the suggested FIF-model.

In Figure 5 the feedback arrows is analogues to the level-4 in the JDL model and since the level-4 is aligned 
with Act this indicates that there are a more versatile structure than just one JDL and OODA-loop.

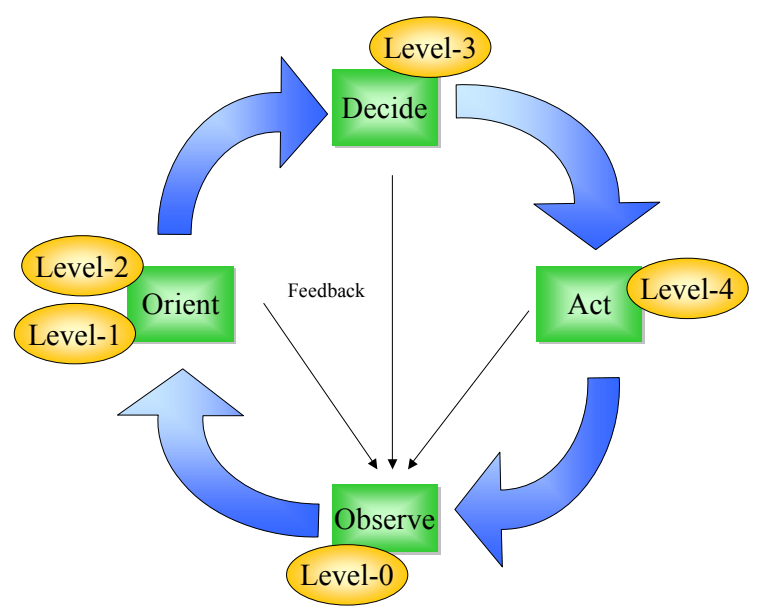

Figure 5: OODA-loop with JDL, according to Bedworth

\subsection{KID}

The Knowledge, Information and Data model (KID) defined in section 2.3.1 (Figure 2) is somewhat consequent with the slide in Figure 6 presented ((van Dam 2004).

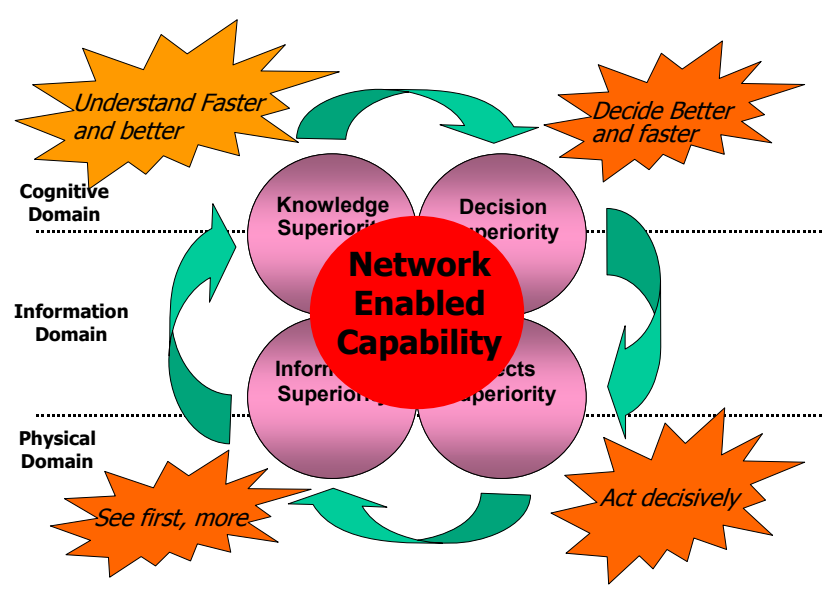

Figure 6: Network Enabled Capability - Domains

The three levels are as follows: place

Physical where the bits and pieces actually takes

Information where the entities are put into context

Cognitive where the human mind start to reasoning

In the KID approach knowledge is a cognitive process, Information is derived data, the physical part is not equal with data but data can beside other representations origin from the physical domain.

Further the KID is adjacent with the Levels of Conceptual Interoperability (LCIM) (Tolk et al. 2003b) visualized in the right side in Figure 7 and are defined as:
No Interoperability no connection is established at all. Data is service specific and no exchange mechanism is established.

Technical physical connectivity is established allowing bits and bytes to be exchanged.

Syntactic data can be changed in standardized format i.e. the same protocols and formats are supported.

Semantic not only data but also it contexts, i.e. information can be expressed using common reference models defining unambiguous meaning of data.

Dynamic information, data that can be expressed through various reference models.

Pragmatic information, data is expressed using reference models. But sometimes the information, data is expressed with other sets in the reference model. For example the sentence "Its raining cats and dogs" is such idiom.

Conceptual a common view of the world is established.

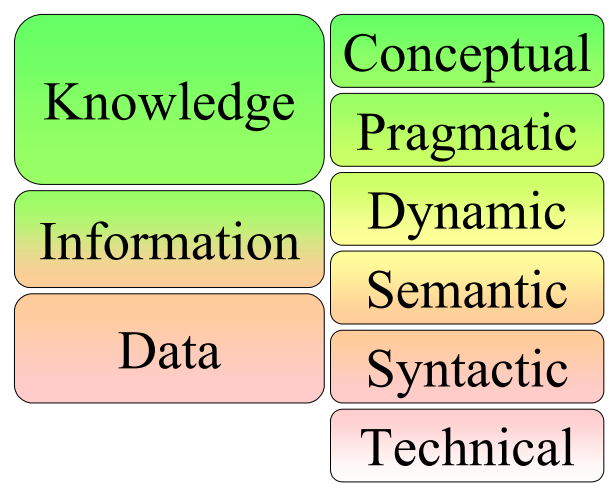

Figure 7: Knowledge Information and Data mapped to LCIM

Within Figure 7 the KID are mapped with the LCIM with the view that data is the unrefined "product" and that data that are washed, fusion through a system becomes Information. This information can then depending on the receiver is treated as data or information. Knowledge is the internal representation of that systems belief made upon the information pieces it has.

\subsection{Temporal situation picture}

"The longer we wait, the more things we will know" The models described so far embrace a process-oriented representation of decision support and information fusion with a hierarchical knowledge representation (fractal in KID). Depending on when in time the data occur the resulting information might differ from the origin assumed situation picture changing the knowledge. Therefore the temporal data, information knowledge aspect is needed to address in decision-making and systems to support the decision makers. Lets for a moment recapitulate the Previous, Present, Predicted model in Error! Reference source not found.. Their data origin from three time modes. 


\section{Gustavsson and Planstedt}

Prediction information shaped from simulations, showing the guessed state

Present information acquisition from sensors, showing the current state.

Previous information retrieved from databases, showing the well-known state.

The situation picture produced is the combination of previous, present and predictions, the validity of the situational picture depends of new observations and predictions that changing and enriching the situational picture. The previous situational picture was still valid for the decision made at that particular time. The new information that overturns the old assumption can also be used in validating the old situational pictures, actually refining he old situational pictures. There is also a dimension in how "fast" the sensor input is to the information fusion process providing the situational pictures. Human Intelligence (HUMINT) report may be available hours or days after the actual incident, giving the "true" picture of what happened. This updated situation picture for the previous time can then be used in estimating how good our present situation picture is. This estimating process is without of scope of this paper but interesting.

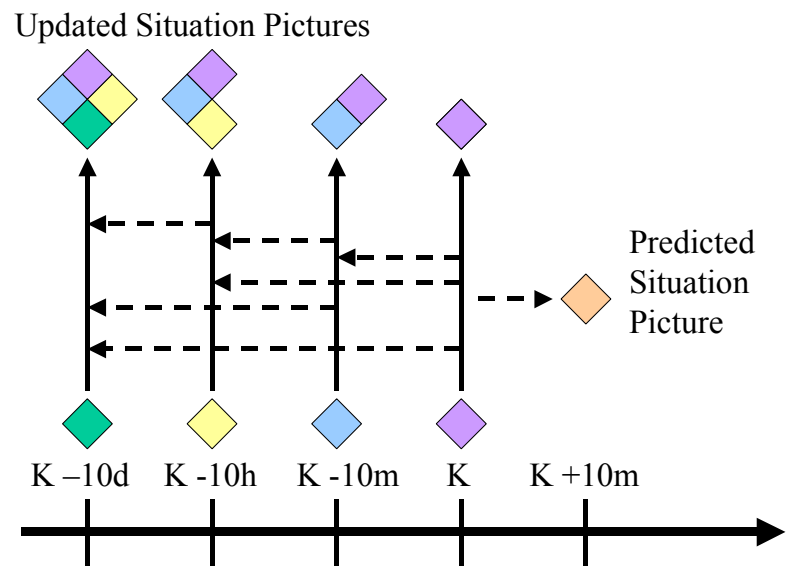

Situation Picture

Figure 8: Temporal Situation Pictures

In an attempt to visualize the Temporal Situation Pictures and the refining is shone in

Figure 8. Observations that occurred in K-10d are fusion with the situational picture origin K-10h, K-10m and $\mathrm{K}$ giving an updated, closer to reality. For K-10h the K$10 \mathrm{~m}$ and $\mathrm{K}$ is used in the fusion process.

The pattern in how the situational pictures are refined over time gives an ability to assess how good the information fusion process is in providing situational pictures.

Compensating transactions can be introduced to adjust the current situational picture. Further the pattern in the new observations gives the ability to predict what information that might occur in the future, enabling for a predicted situational picture.

\subsection{The FIF model}

The Fractal Information Fusion OODA (FIF) presented here is a fractal model incorporating the OODA-loop, JDLmodel, KID and P3IF. One of the arguments for a FIFmodel is that there is not only one decision process executing in a humans mind there is plenty. The parts in the OODA-loops then consist of smaller OODA-loops.

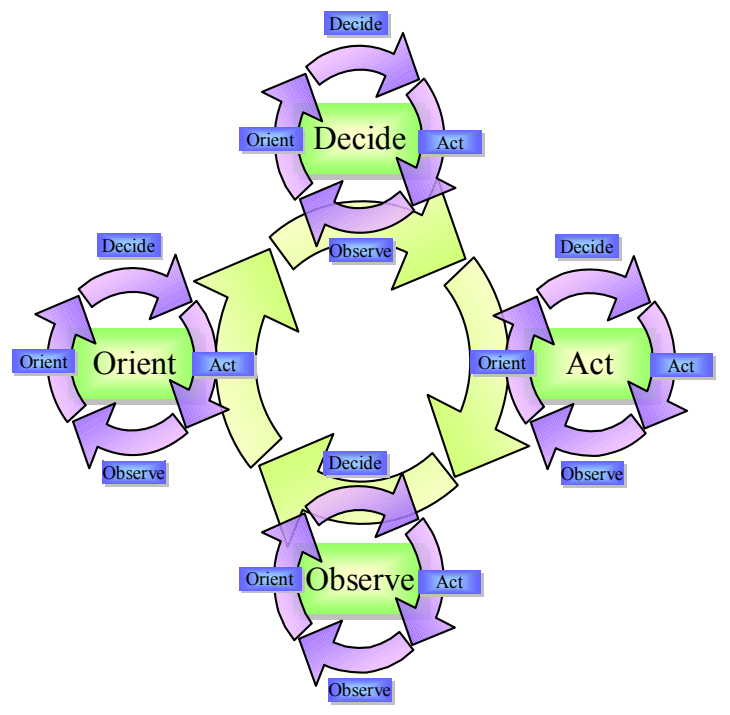

Figure 9: Multiple OODA-loops

Expanding the reasoning that there exist OODA-loops in all four steps of an OODA-loop into a simple example. Consider that a decision maker gives an order to a sensor unit to deliver information to the decision makers C3I system. The decision-maker is in the Act phase the receiving commander is in his or hers observe, the actual sensor system, able to delivering information is un-linked at the moment. The sensor commander orients conduct some decisions from the action matrix, continuous loops. Then the sensor system starts to deliver information to the C3I system, the sensor system is in Acting phase. The decision maker receives this information in his or hers Observe phase. The sensor system is then a part of the Observe phase of the decision maker. Still the sensor system has its own OODA-loop, its own JDL hierarchy, its own data retrieval systems and a Kalman filter (another JDL, OODA-loop). The OODA-loops are connected in multiple (Figure 9).

So using the mapping visualized in Figure 5 there exists complete JDL-models in each OODA-loop. Enabling that a system, or sub-system, that have an OODA-loop also have a companion JDL-model mapped. In the Systems-ofSystems thinking this model is more accurate since allows to have own JDL-model view, own OODA-loop visualized in the same context enabling reasoning in how to build systems that can live in such wide-ranging world. The KID approach as well as the time view is conformant with both 
the JDL-model and OODA-Loop. The steps within the JDL-model, Object, Situation and Intention maps to the data, information and knowledge since the both express a knowledge refinement hierarchy that is fractal. The combination of the four, (OODA, JDL, KID, P3IF) are illustrated in Figure 10 (where the P3IF's Present part is exemplified).

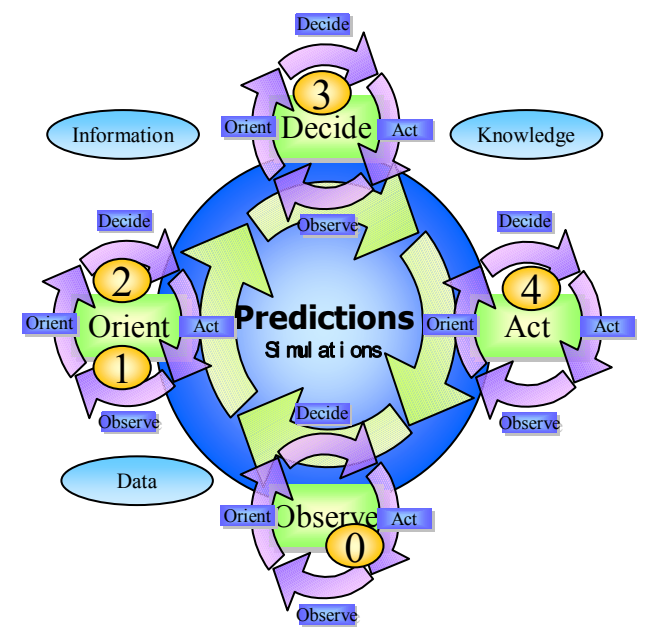

Figure 10: Fractal OODA, JDL, KID and part of P3IF

The fulfillment of the FIF-Model is that each part in FIF consists of a FIF-model on a higher, lower or subsidiary level (Figure 11). To illustrate the fractal behavior in the picture reduces the easy visualization so the intention with figure 10 and figure 11 is to give an overall picture not a detail picture. The purpose is to show that the four models coexist and that the FIF-model is a mediator between the various domains.

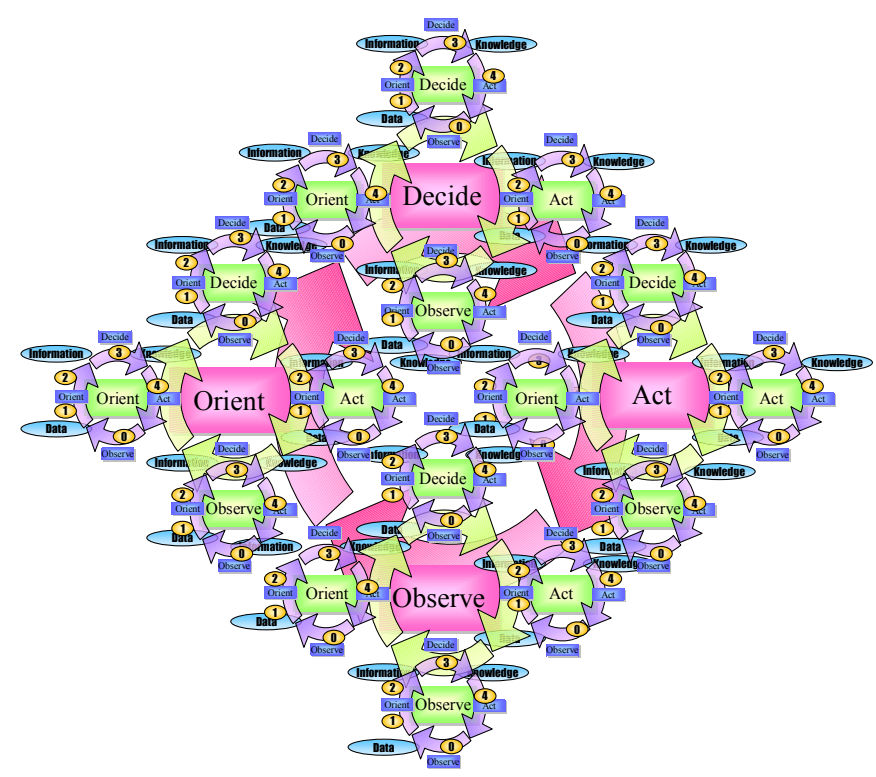

Figure 11: The Fractal Information Fusion OODA Model (FIF)
With the FIF-model at hand the next question is in how to compose an architecture that are valid at all levels in the FIF-model.

\section{MULTI-HYPOTHESIS INTENTION SIMULATION AGENTS ARCHITECTURE}

In the paper (Gustavsson et al. 2005a) the MuHISA architecture is described in more detail and here the overall architecture is described. The architecture is based on the FIF-model, Service Oriented Simulations (Gustavsson et al. 2004; Gustavsson et al. 2005b), Active Database, Agents, Current work with C-BML (Tolk et al. 2004; Blais et al. 2005) and GIG, FMA, NNEC (United States. Joint Chiefs of Staff.; United States. Joint Chiefs of Staff. 2000; Defense 2003; Defense Material Administration 2003; Öhlund et al. 2003; Buckman 2004; van Dam 2004). The FIF-Model is presented in this paper and the Service Oriented Simulation is the combination of the FMA reference implementation "Open Services Infrastructure" and adding simulation specific services upon it. The Active Database approach is to enable for Event Condition Action (ECA) rules enabling for reactive behavior upon internal states in the database. Agent technology enables self-configuration and the ability to find new relations dynamically. The CBML work is essential to provide unambiguous representation of intention for systems interchanging information between C4I systems, Simulations and robot forces. Finally the GIG, NNEC, FMA sets the system-of-systems view and the environment where a MuHISA application shall exist.

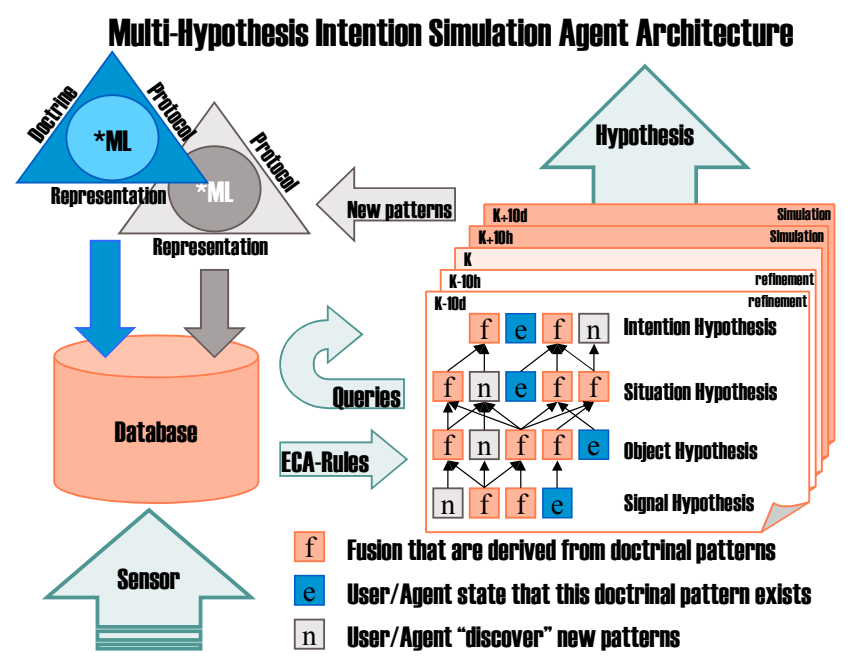

Figure 12: MuHISA Architecture (relaxed)

The walk-through of the architecture in Figure 12 starts with the "star"-ML in the upper left corner. The "star"-ML is a Management language that provides a grammar for a doctrine to be expressed with supporting Information Ex- 


\section{Gustavsson and Planstedt}

change Data Model and transport protocol assigned enabling for easy integration. The management language holds the information of When, Why, What, Who, Where (5W) and are instantiated in the database where the IEDM provides with a structure of object-types, object-items, actions and so forth. The doctrinal relations are then kept in the database enabling for queries to be asked if monitored data supports the various doctrinal relation constructs (patterns).

Sensor data is constantly feed to the database using an agreed Information Exchange Data Model. Queries ca is asked using simple SQL queries or use data-mining techniques. The ECA-rules provides for rule based dynamic behavior where agents can be started as a consequence of the trigging event. The various data elements needs to have operations such as equal, greater, lesser implemented to support the aggregation and querying.

In the picture five different epochs are showed two from previous one present and two predictions and depending in which focus the actual system has this are adjust to a sufficient number. The level of Hypothesis generating is also a design issue to take into account. The Predictions uses simulation to realize sensor input. The three agents present are f: fusion, e: exist and n: new.

$\mathrm{F}$ is fusion made from the doctrinal knowledge represented in relations in the database providing for patterns.

$\mathrm{E}$ is the existing doctrinal patterns that have not been detected by lower level fusion agents. But a user or agent decides that this belief is present.

$\mathrm{N}$ is the agents searching for new relational constellations between the data representations (objects) in the database.

The resulting outcome is a continuously series of hypothesis for the various levels (Signal, Object, Situation and Intention).

The implementations of the agents/models are not forced to be fixed. The internal representation of knowledge is up to the agent architecture. In (Wooldridge et al. 1995) some architectures are presented however the main issue is to provide that agents, if they want to, can communicate and be interoperable. The actual simulation models fidelity is not in the scope of this paper.

\section{SUMMARIZATION}

In the development of architecture for hypothesisgenerating questions about how the system looks like arises how does decision models look like and information fusion models look like, what is knowledge and so forth. In the work the starting point was the traditional OODA-loop and JDL-model system theories, the ongoing work with GIG and similar approaches. Either the OODA-loop or the JDL-model is sufficient enough to express system-of.systems thinking. With the suggested FIF-model the system-of-system thinking is possible to express and the development of a generic architecture for information fusion is then possible to build. Un the ongoing work with $\mathrm{Mu}$ HISA architecture the reasoning using the FIF-model has been fruit full.

The FIF-model is not a complete model and the ongoing work with MuHISA Architecture and its reference implementation will most certainly challenge the FIF-model.

A further work is to align the FIF-model with Lagervik-Situational Awareness-model (lagervik 2005) based on Activity Theory (Bedney et al. 1999) and Adaptive Learning Management system (Ackoff 1999).

\section{ACKNOWLEDGMENTS}

The authors want to acknowledge Mr. Pelle Johannisson for his never-ending creative mind blowing odysseys. And not to forget our co-workers and companions at Ericsson, University of Skövde, Swedish Defence Material Administration and Swedish Armed Forces.

\section{REFERENCES}

Ackoff, R. L. 1999. Ackoff's best: his classic writings on management John Wiley \& Sons, New York, 1999.

Andler, S. F., L. Niklasson, B. Olsson, A. Persson, T. Planstedt, L. J. d. Vin, B. Wangler, and T. Ziemke 2004. Information Fusion from Databases, Sensors and Simulations, University of Skövde, SE-54128 Skövde, Sweden, Skövde, 2004.

Bedney, G., and D. Meister 1999. Theory of Activity and Situation Awareness, International Journal on Cognitive Ergonomics (3) 1999, pp 63-72.

Blais, C., K. Galvin, and M. Hieb 2005. Coalition Battle Management Language (C-BML) Study Group Report, Fall Simulation Interoperability Workshop, Orlando, FL, US

Boyd, J. 1986. Patterns of Conflict, 1986.

Buckman, T. 2004. Network Enabled Capability Feasibility Study, MILCOM 2004

Defense, D. o. 2003. Global Information Grid (GIG) Overarching Policy, 8100,1.

Defense Material Administration 2003. IRM-Vision, rapport från MS 850 AO 610530.

Försvarsmakten Högkvarteret. 2003. Målbild för utveckling av försvarsmaktensledningssystem 2010) 2003.

Gustavsson, P. M., L. Nikalsson, and P. Ericsson 2005a. Multi-Hypothesis Intention Simulation Agent Architecture -MuHISA, to be submitted to BRIMS-06, Skövde

Gustavsson, P. M., T. Planstedt, Å. Björk, and C. Brax 2004. Towards Service Oreinted Simulations, Fall iSimulation Interoperability Workshop 2004, Orlando, FL, US

Gustavsson, P. M., T. Planstedt, G. Karlsson, C. Brax, and Å. björk 2005b. Service oriented Simualtions Concept, SimTecT, Sydney, Australia, pp. 233-238. 
Kock, J. 2005. Computer supported simulation from a learning perspective) 2005.

lagervik, C. 2005. A System Theoretical approach to Situation Awareness - A holistic view of purposeful elements, in: School of Humanities and Informatics, University of Skövde, Skövde, 2005, p. 52.

McDaniel, D. 2002. Multi-Hypothesis Database for LargeScale Data Fusion, Information Fusion

Pioch, N. J., D. Hunter, J. V. White, A. Kao, D. Bostwick, and E. K. Jones Multi-Hypothesis Abductive Reasoning for Link Discovery,

Schuck, T. M., B. J. Hunter, and D. D. Wilson 2004. Multi-Hypothesis Structures, Taxonomies and Recognition of Tactical Elements for Combat Identification Fusion, 9th International Command and Control Research and Technology Symposium, Copenhagen, Denmark

Steinberg, C., Bowman, and F. White 1999. Revisions to the JDL Data Fusion Model, Proceedings of SPIE, Sensor Fusion: Architectures, Algorithms, and Applications III

Tolk, A. 2004. An Agent-Based Decision Support System Architecture for the Military Domain IOS Press B.V., 2004.

Tolk, A., K. Galvin, M. Hieb, and L. Khimeche 2004. Coalition Battle Management Language, Fall Simulation Interoperability Workshop, SISO, Orlando, Florida

Tolk, A., and M. R. Hieb 2003a. Building \& Integrating M\&S Components unto C4ISR Systems for Supporting Future Military Operations, 2003 International Conference on Grand Chalanges för Modeling and Simulation (ICGCMS'03), p. 13.

Tolk, A., and J. A. Muguira 2003b. The Levels of Conceptual Interoperability, Fall Simulation Interoperability Workshop, SISO, Orlando, Florida

Tolk, A., and M. J. Pullen 2003c. Ideas for a common Framework for Military M\&S and C3I Systems, 2003 Euro Simulation Interoperability Workshop, SISO, Stockholm

United States. Joint Chiefs of Staff. Joint Vision 2010 [online]. Available via http: / / www.dtic.mil/jv2010/jv2010.pdf

United States. Joint Chiefs of Staff. 2000. Joint Vision 2020, Joint Chief of Staff, Washington, D.C.

van Dam, R. S. 2004. Transforming the NATO alliance: The Challange of NATO Information Superiority \& Network Enabled Capability, Industry Day 2004 NATO's Transformation Command "A Partnership with Industry"

Wooldridge, M. J., and N. R. Jennings 1995. Agent Theories, Architectures, and Languages: A Survey, Workshop on Agent Theories, Architectures \& Languages, Springer-Verlag, Amsterdam, The Netherlands, pp. 1-22.
Öhlund, G., J. Bendz, P. Johannisson, and P.-G. Jönsson 2003. The Swedish Armed Forces Enterprise Architecture, 03E-SIW-049.

\section{AUTHOR BIOGRAPHIES}

PER M. GUSTAVSSON is a research engineer working with advanced decision support systems at Ericsson Microwave Systems AB, Security Solutions. He is also an industrial Ph.D. student at University of Skövde, Sweden enrolled at De Montfort University, Leicester, UK. He has over ten years of professional software development knowledge. Gustavsson holds a M.Sc. in Computer Science, New Generations Representations, Distributed RealTime Systems and a B.Sc. in Systems Programming both from University of Skövde, Sweden. His e-mail address is per.m.gustavssonaericsson. com.

TOMAS PLANSTEDT is a product manager working with advanced decision support systems within security solutions at Ericsson Microwave Systems AB, Security Solutions (EMW). He is in charge for Ericssons' part in the Information Fusion research project with University of Skövde. Planstedt has over ten years of professional software development knowledge. Planstedt holds a B.Sc. in Computer Science from University of Skövde, Sweden. His e-mail address is tomas.planstedt@ericsson.com. 